\title{
Neuropsychobiology 1986;15:220
}

\section{Subject Index}

Affective disorders 57 Age psychosis 122 Aggressive behaviour 133 Alzheimer's disease 130,133 Amitriptyline 15, 73, 146 Anorexia nervosa 89 Antidepressant pharmacotherapy, children 143

- treatment 62

Artifacts detection 208

Attention 165

Autism, infantile 160

Automatic processing 89

Autoregressive processes 208

Basal ganglia 13 Behavior 57, 165

- test 95 Biogenic amines 95 Biotransformation 15 Brain nucleic acids 130

regions 95

Capacity of structurizịng 34

Caries activity 143

Catecholamine hypothesis 57

Chorea 13

Clinical status 49

Corticotropin, hormonal action 165

Cortisol 84

Critical flicker frequency 187

Daytime sleeping 187

Dementia 133

Depression 109

Depressive patients 15

Desmopressin 80

Dexamethasone suppression test 68,109

Diazepam 29

Digit symbol substitution test 187

4-Dihydroxyphenylethyleneglycol, plasma

62 3,4-Dihydroxyphenylethyleneglycol, plasma

68 Drug action model 192

Efficacy 160 Effortful processing 89 
Electroencephalography 49, 165

Enuresis 143

Epidemiology 122

Erythrocytes 7

Ethanol 29

Event-related potential 201

Evoked potentials 165

Exercise 84

Experimental design 192

Factor analysis 49 Fear conditioning 29 Frequency analysis 101

Growth hormone 84

Incidental learning 89

Indalpine 62

Isopropyl barbiturate derivatives 95

Learning 192

Lithium prophylaxis 34

Long-time electroencephalogram 208

Lundby|study 122

Major depression 62,68 Maprotiline 62, 73, 146 Membrane transport 7 Memory 29,80, 192 - molecules 130 3-Methoxy-4-hydroxyphenylglycol

57 Methylphenidate 84 Multi-infarct dementia 122

N100 201

N120 evoked potential 1 Neuroleptics 160 Neuropeptide 114 Neuropeptides 165 Nootropic drugs 192 Norepinephrine 57

Overlapping memory codes 130

Paired associate learning 43 Phenothiazine 15 Phenytoin 201 
Plasma prolactin level 155 Platelet monoamine-oxydase 43 Prolactin 84 Propranolol 20

in psychiatric therapy 20

Psychiatric side effects 20

Psychopathology 57

Psychosis 13

Psychostimulant drugs 84

Radioelectroencephalogram 101 Rapid information processing 187 Robust filters 208 Routine and nonroutine processes 34

Saliva composition 73,146

secretion 73

- - rate 146

Sandoz clinical assessment - geriatric 49 Schizophrenia 1, 109, 114 Senile dementia 122

- dementias 130 Sensation seeking 43 Serotonin (5-HT) 133

- metabolism 95

Serum dopamine $\beta$-hydroxylase 43

Simulated shift work 187

Spectral analysis 49

Stimulatory drugs 101

Stress 84

Subjective sleep quality 187

Sulpiride 155

Tachistoscopic testing 34 Temazcpam 187

Thyrotropin-relcasing hormone 114 Tryptophan 133 L-Tryptophan 7 L-Tyrosine 7

VasOpressin 80, 114 Visual perception 34

Zimelidine 29,73, 146

Supplement 1 has its own subject index. 